\title{
Dental Bleaching Using Violet Light Alone: Clinical Case Report
}

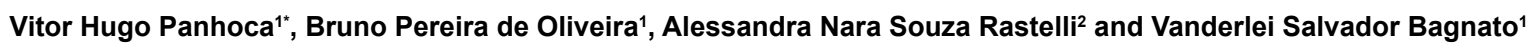

${ }^{1}$ São Carlos Institute of Physics, University of São Paulo, PO Box 369, 13560-970, São Carlos, SP, Brazil

${ }^{2}$ Department of Restaurative Dentistry, Universidade Estadual Paulista, Humaitá St 1680, Araraquara, SP, Brazil

\begin{abstract}
In dental offices, one of the most frequent activities is dental bleaching. The evolution of protocols and the possibility of combining the use of light with chemical products can be found in the literature. Some articles only describe using light with a whitening agent. This is a topic of great interest and this article shows a clinical case report using violet light applications. The final results were evaluated over 2 months to assess color stability and verify that they do not show great variation.
\end{abstract}

Keywords: Dental bleaching; Violet light; Colorimetric

\section{Introduction}

The first reports of dental bleaching appeared in 1872 when oxalic acid was used. After this, other publications and patents on this subject could be found [1,2]. However, it was the 1991 publication by Haywood \& Heymann which used $10 \%$ carbamide peroxide that popularized the home dental bleaching technique in living teeth [3]. Since then, various techniques using peroxide-containing gel for home use or inoffice have been developed to improve this procedure and seek a better result in obtaining more efficient dental bleaching, absence of dentin hypersensitivity and greater color stability after treatment.

Bleaching is a clinical procedure that provides better dental and overall facial aesthetics to patients who go to the dentist's office frequently. Currently, this treatment uses carbamide peroxide or hydrogen peroxide for applications at home or in-office, usually or even by combining these two techniques In-office procedures can be carried out using blue light $(450 \mathrm{~nm})$ combined with peroxides in order to obtain whitening faster and this is a widely used technique [4].

Recently, a new device using violet wavelengths (approximately $410 \mathrm{~nm}$ ) has been developed. The results of the bleaching shown are better than the traditional protocol [5]. The main characteristics of this new technique were the observation of bleaching without gel (such as peroxides) and the absence of dentin hypersensitivity in treated patients. Violet light has more energy carried in its photons and is more absorbed on the dental surface due to its physical characteristics. Reports from the literature justify that these physical violet light characteristics are responsible for larger surface absorption of light and breaking the pigments (bleaching) that stain teeth without needing to use hydrogen peroxide containing a high concentration of whitening agent gels [5-7].

In addition, using violet light in a fractional way, as presented in this communication, prevents heating of the tooth structure [8]. Therefore, in the technique recommended using violet light in a fractional manner and without using a peroxide-containing gel, efficient dental bleaching without producing dentin hypersensitivity can be achieved $[7,8]$. The combination of home and in-office bleaching techniques is advocated in the literature because they show greater color stability to treated teeth [9]. Thus, in an attempt to obtain greater color stability after bleaching using violet light without gel, we present a clinical case treated using violet light and then mineral oil. The color stability was evaluated afterwards.

\section{Clinical Case}

After signing an informed consent form agreeing to dental bleaching treatment, a 27-year-old male patient was treated in-office at the Biophotonics Laboratory at the Institute of Physics São Carlos (IFSC in Portuguese) at the University of São Paulo (USP). A radiographic examination was performed and normal and complete rhizogenesis of 11 and 21 was found. The patient was advised to receive in-office dental bleaching using a device with LED emitting violet light (BrightMax Whitening System-BMW, MMoptics, São Carlos, SP, Brazil) without using peroxide-containing bleaching gel. Figure 1 shows the wavelength of violet light used, whose maximum emission peak is $408 \pm 10 \mathrm{~nm}$. The device emitting violet light had an estimated total power of $1400 \mathrm{~mW}$ and surface contact irradiance of $165 \mathrm{~mW} / \mathrm{cm}^{2}$.

To document the results, a photograph of the clinical case was taken before and after so that they could be compared. All clinical procedures were performed using a lip retractor. The initial color of the upper and lower teeth was obtained immediately after prophylaxis using a

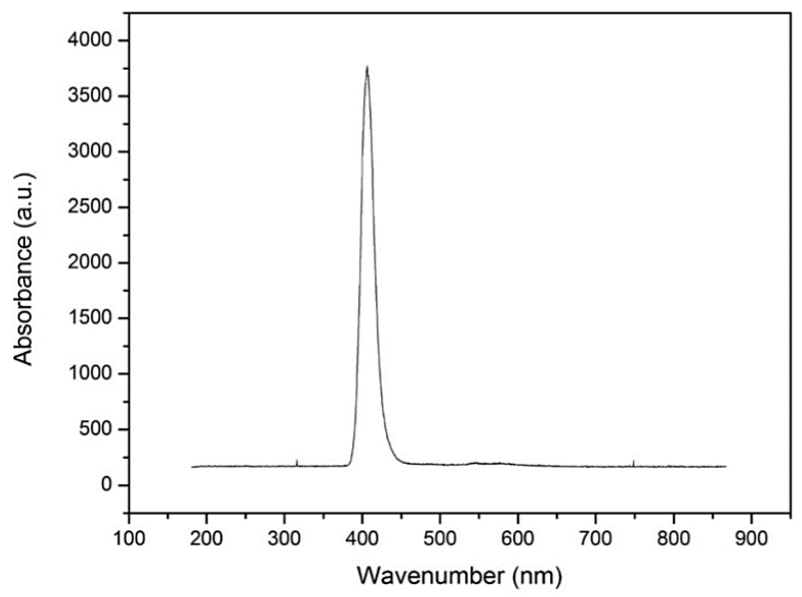

Figure 1: Violet LED emission spectrum.

*Corresponding author: Vitor Hugo Panhoca, São Carlos Institute of Physics, University of São Paulo, PO Box 369, 13560-970, São Carlos, SP, Brazil, Tel: 0049613142111; E-mail: vhpanhoca@ifsc.usp.br

Received August 23, 2017; Accepted September 28, 2017; Published October 05, 2017

Citation: Panhoca VH, Oliveira BPde, Rastelli ANS, Bagnato VS (2017) Dental Bleaching Using Violet Light Alone: Clinical Case Report. Dentistry 7: 459. doi:10.4172/2161-1122.1000459

Copyright: (c) 2017 Panhoca VH, et al. This is an open-access article distributed under the terms of the Creative Commons Attribution License, which permits unrestricted use, distribution, and reproduction in any medium, provided the original author and source are credited. 
Citation: Panhoca VH, Oliveira BPde, Rastelli ANS, Bagnato VS (2017) Dental Bleaching Using Violet Light Alone: Clinical Case Report. Dentistry 7: 459. doi:10.4172/2161-1122.1000459

Robson brush and pumice paste diluted in water with a low-rotating instrument using a Vitapan Classical color scale (Vita Zahnfabrik, H. Rauter GmbH \& Co. KG.D-7880 Säckingen, Germany) to compare the final results with the initial color prior to treatment (Figure 2). The color of the treated teeth was also recorded using a colorimetric spectrophotometer (Pocket Spec ${ }^{\bullet}$ - PocketSpec Technologies Inc - Denver, CO - USA) which quantifies the color changes so that the dental bleaching treatment can be compared before and after treatment with numerical quantities.

After the initial color was recorded, we evaluated the degree of dental sensitivity presented by the patient using an air jet from a triple and tactile syringe applied on the cervical third of all dental elements. Dental bleaching was started with the violet light system (BMW, MMoptics, São Carlos, SP, Brazil), maintaining the active end of the LED device as close as possible to the dental surface. The operation takes place in cycles of 60 seconds (on) and 30 seconds (off) (Figure 3). The upper and lower arches were irradiated with violet light simultaneously. Irradiation was repeated 20 cycles performing a total illumination time of $20 \mathrm{~min}$ and a total procedure time of $30 \mathrm{~min}$. After applying the light in each session, we then used mineral oil (Nujol) by rubbing the vestibular surface of each tooth for 10 seconds.

The clinical session was concluded with prophylaxis carried out using a Robson brush with pumice paste diluted in mineral oil. In this clinical case, we performed three sessions with an interval of one week between each. The result of the dental bleaching obtained in the first session can be seen in Figure 4. The patient reported having no dental sensitivity during and after the violet light applications, which was reported in all sessions. After the 3 dental bleaching sessions, the color (Figure 5) and sensitivity were evaluated and the evaluation procedure was repeated 1 week, 30 days and 60 days later, respectively (Figure 6).

\section{Quantitative Analysis of the Whitening Process}

For the quantification of the changes promoted by the procedure, we measured the RGB components using a colorimetric spectrophotometer (Pocket Spec ${ }^{\oplus}$ - PocketSpec Technologies Inc - Denver, CO - USA) where the color components $\mathrm{L}, \mathrm{a}, \mathrm{b}$ are obtained. To be considered standard white, the components are L1, a1 and b1. These components determine a three dimensional vector $\mathrm{v} 1$ in the RGB coordinates (Figures 7 and 8).

The measured sample components compose a second vector v2 (with components L2, a2 and b2). As much as v2 approaches to v1, closes to the standard white is the sample. The magnitude of vector $\mathrm{E}=\mathrm{v} 1-\mathrm{v} 2$ quantities how distant from the standard white the sample is. Therefore, we used the quantity: $/ E /=\sqrt{ }(L 1-L 2)^{2}+(a 1-L 2)^{2}+(b 1-b 2)^{2}$

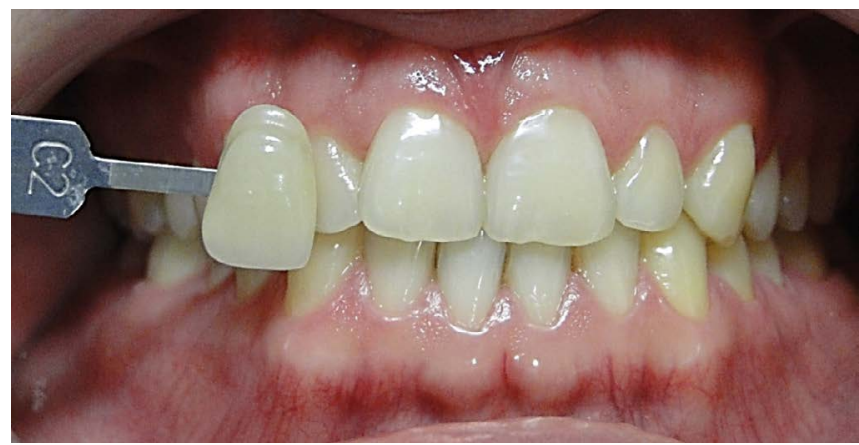

Figure 2: Photograph showing the color of teeth in C2 on Vitapan classical guide.

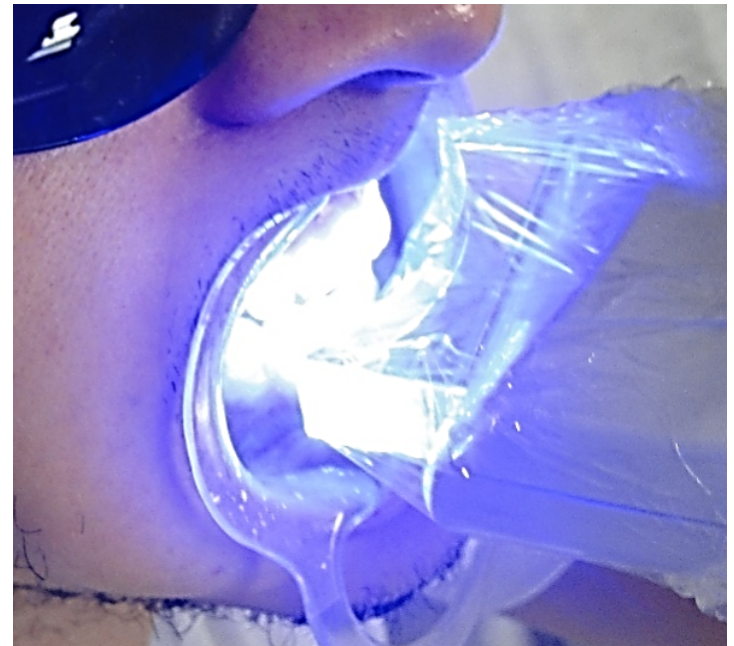

Figure 3: BMW device (MMoptics, São Carlos, SP, Brazil) applying violet light on the teeth.

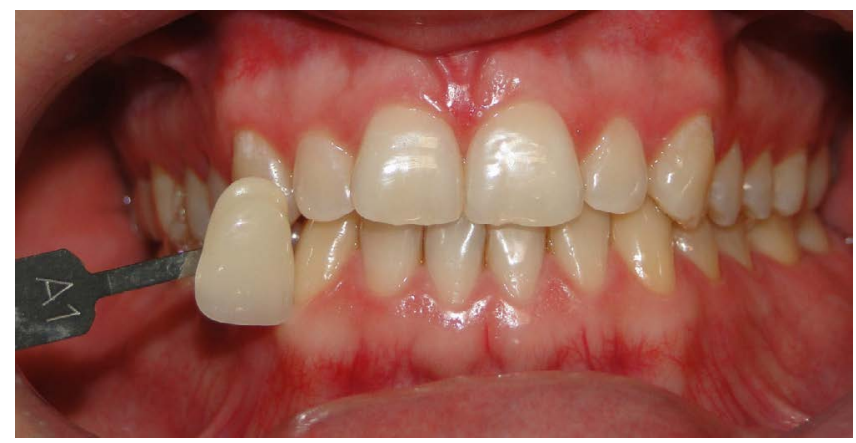

Figure 4: Final photo after the 1st session showing the color of the teeth in A1.

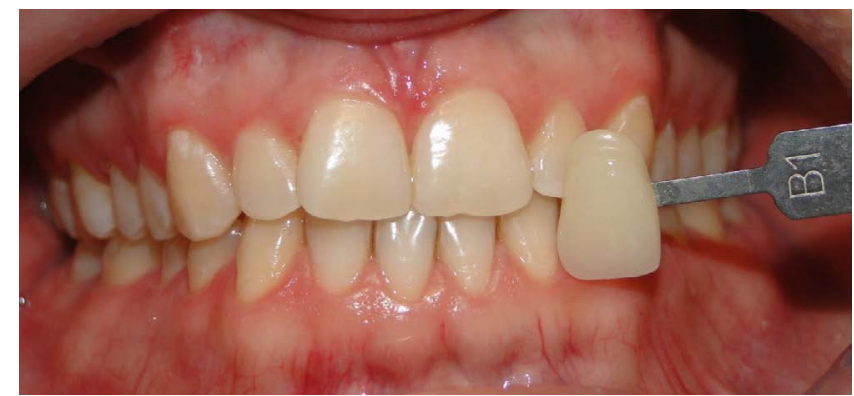

Figure 5: Final photo after the 3rd session showing the color of the teeth in B1.

to measure the approximation to standard white. Smaller value of $\mathrm{E}$ represents better achieved whitening.

\section{Results}

The evolution for the results of dental bleaching using violet light can be seen in Table 1, which shows a decrease in oscillation and stabilization in the color on the Vita scale after treatment. It can be observed that after the clinical sessions, the initial color of the treated 

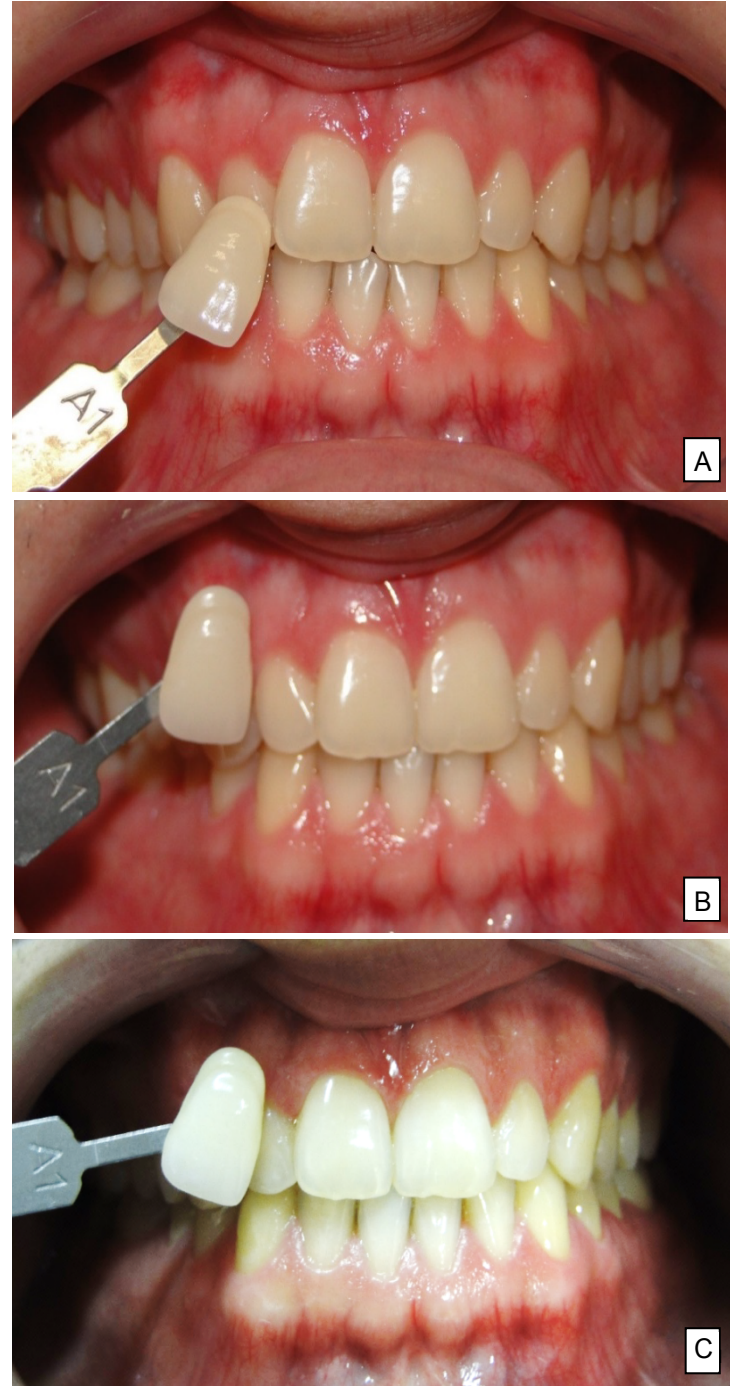

Figure 6: Final photos after 1 week (A) with color $A 1,30$ days (B) with color $\mathrm{A} 1$ and 60 days $(\mathbf{C})$ with color $\mathrm{A} 1$.

\begin{tabular}{|c|c|c|c|c|}
\hline \multirow{2}{*}{ First protocol } & & Vita colors & \multicolumn{2}{|c|}{ Sensibility } \\
\hline \multirow{2}{*}{ Second protocol } & Initial & C2 & 0 & Air \\
\cline { 2 - 5 } & End & A1 & 0 & 0 \\
\cline { 2 - 5 } & Initial & B2 & 0 & 0 \\
\hline \multirow{2}{*}{ Third protocol } & End & B1 & 0 & 0 \\
\cline { 2 - 5 } & End & A1 & 0 & 0 \\
\hline \multirow{2}{*}{ After 1 week } & ---- & A1 & 0 & 0 \\
\hline After 1 month & ----- & A1 & 0 & 0 \\
\hline After 2 month & & A1 & 0 & 0 \\
\hline
\end{tabular}

Table 1: Qualitative analysis of the dental bleaching.

teeth was $\mathrm{C} 2$ and became $\mathrm{A} 1$ at the end. Table 1 also shows the absence of pain sensitivity throughout and after treatment.

In Figure 8, a graph showing the results measured by the spectrophotometer confirming the results obtained with the Vita scale can be seen.
The results are normalized to indicate dental bleaching in each protocol, which after each procedure verifies this decrease in the Euclidean distance. This shows that bleaching is always achieved and stabilization is observed until the end of the color evaluation after 60 days.

\section{Discussion}

For the sake of comprehension and understanding the discussion of this subject, contextualizing the topic, dental bleaching using gel containing peroxide or combined with light is a dental procedure that, in many cases, has undesirable side effects and includes dental decalcification and dental sensitivity [10-12]. Much research conducted on dental bleaching has been developed to inhibit these side effects $[10,13]$. Most of these effects are caused by the chemical bleaching agent. Another problem encountered after dental bleaching is the lack of color stability. Some authors advocate combining in-office bleaching with a home bleaching technique with trays using peroxide gels at a low concentration aiming to obtain greater tooth whitening longevity [9].

In addition to its wide use, chemical substances in dental bleaching are sometimes questioned due to the side effects of superficial decalcification of tooth enamel and promoting dental sensitivity due to the production of nascent oxygen that is responsible for breaking the pigments that stain the teeth $[10,13]$. Many authors justify that

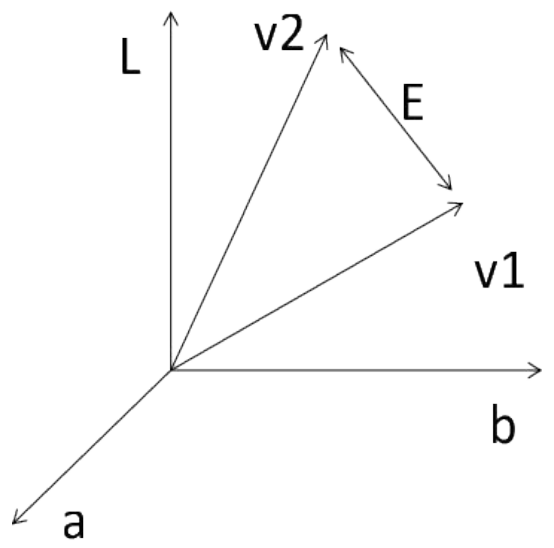

Figure 7: Analysis in Euclidean space graphics.

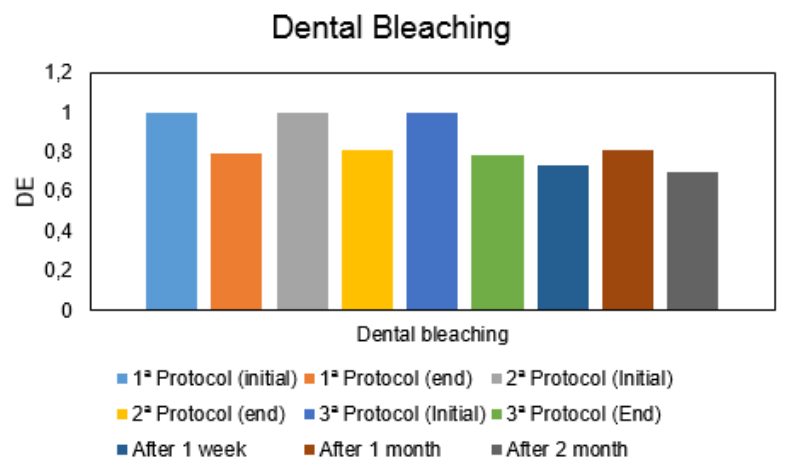

Figure 8: Result of dental bleaching during the whole experiment. 
remineralization after dental bleaching gel re-establishes after some days by the calcium found in the saliva or even recomposes when fluoride gel is applied after bleaching [14].

Nascent oxygen has a low molecular weight and diffuses through the enamel prisms and dentinal canaliculi allowing for sensitization of nerve terminals of the dental pulp that extends to the dentin causing dental sensitivity [15]. Using light in dental bleaching is justified to accelerate the tooth whitening effect, but is contested by some authors who believe it is unnecessary to use it for the activation of this nascent oxygen formation reaction [16]. In addition, using blue light carelessly for dental bleaching can lead to dental heating producing thermal damage to the dental pulp and painful sensitivity [17].

Violet light used for dental bleaching appears as a new alternative in dental treatments because of its isolated use without needing to use peroxide gels presenting physical properties capable of breaking pigments and obtaining dental bleaching [6-8]. Violet light has the characteristic of not having a demineralization effect on the teeth and when used in a fractional way with intervals of time without continuous application does not cause heating of the teeth, which makes this technique efficient without causing sensitivity [18].

The color stability of in-office dental bleaching was questioned not only when combined with peroxide gel, but also when using only violet light. For this reason, many authors argue that after in-office dental bleaching, maintenance with home bleaching should be done using low concentration peroxide gels or even whitening toothpastes [19]. The results presented in this article show a technique that combines using mineral oil (Nujol) after dental bleaching with violet light to obtain the removal or prevent the reconnection of broken pigments present in the enamel and dentin and to promote greater stability in this technique. Mineral oil works as a remover that binds pigment molecules broken by violet light and removes them giving greater effectiveness and stability to dental bleaching.

The clinical case presented shows that the dental bleaching done by applying violet light promoted a decrease in color in the Vita scale of the treated teeth. Before and after treatment can be observed in the photographs where the initial color in the Vita color scale shows C2 and $\mathrm{B} 1$ at the end of the third clinical session. It can be observed that after 1 week of finishing dental bleaching, the color of the teeth presented in the Vita scale became a little darker, showing color A1. This A1 color scale was maintained after 60 days (Figure 6a-6c). A dental pain sensitivity test was carried out on the patient before and after the treatment using an exploratory catheter and air. The patient reported complete absence of dentin hypersensitivity during and after dental bleaching, as can be seen in Table 1.

Color stability is usually not easily achieved with only light bleaching. We hypothesized that the application of mineral oil to the dental surface may penetrate the first layer of the tooth structure, preventing further recombination of pigment molecules. However, more in-depth investigations must be carried out in this hypothesis

New studies should be carried out in order to obtain better results in dental bleaching using violet light to obtain optimization in the clinical care time and stability in dental bleaching in the long run.

\section{Conclusion}

For this case report, it can be concluded that violet light (approximately 410nm) presents physical characteristics capable of promoting dental bleaching without combining gel with peroxides. This only-light-bleaching has many advantages and combined with stabilizing greats may lead to long term results.

\section{Acknowledgements}

The authors acknowledge the support provided by: FAPESP (São Paulo Research Foundation) - grant number: 2013 / 07276-1 (CEPOF - CEPID Program).

\section{References}

1. Matis BA, Gaiao U, Blackman D, Schultz FA, Eckert GJ (1999) in Vivo Degradation of Bleaching Gel Used in Whitening Teeth. J Am Dent Assoc 130: 227-235.

2. Goldstein RE, Garber D (1995) Complete Dental Bleaching. Quintessence: Chicago, pp: 28-31.

3. Haywood VB, Heymann HO (1991) Nightguard vital bleaching: how safe is it? Quintessence Int 22: 515-523.

4. Ontiveros JC (2011) In-office vital bleaching with adjunct light. Dent Clin North Am 55: 241-253.

5. Panhóca VH, de Oliveira BP, Bagnato VS (2015) Dental bleaching efficacy with light application: In vitro study. Photodiagnosis Photodyn Ther 12: 357.

6. Zanin F (2016) Recent Advances in Dental Bleaching with Laser and LEDs Photomed Laser Surg 34: 135-136.

7. Dias A, Lago N, Dandara W, Ferreira R, Furtado GS (2017) Photodiagnosis and Photodynamic Therapy Dental bleaching with the use of violet light only Reality or Future? Photodiagnosis Photodyn Ther 17: 124-126.

8. Panhóca VH (2017) Clareamento dental: nova perspectiva para a Odontologia Estética. Rev da APCD 1: 95-101.

9. Giachetti L, Bertini F, Bambi C, Nieri M, Russo DS (2010) A randomized clinical trial comparing at-home and in-office tooth whitening techniques: A nine-month follow-up. J Am Dent Assoc 141: 1357-1364.

10. Kossatz S, Martins G, Loguercio AD, Reis A (2012) Tooth sensitivity and bleaching effectiveness associated with use of a calcium-containing in-office bleaching gel. J Am Dent Assoc 143: 81-87.

11. De Oliveira R, Paes Leme AF, Giannini M (2005) Effect of a carbamide peroxide bleaching gel containing calcium or fluoride on human enamel surface microhardness. Braz Dent J 16: 103-106.

12. Azrak B, Callaway A, Kurth P, Willershausen B (2010) Influence of bleaching agents on surface roughness of sound or eroded dental enamel specimens. $J$ Esthet Restor Dent 22: 391-399.

13. Li Y (2011) Safety controversies in tooth bleaching. Dent Clin North Am 55 255-263.

14. Heshmat H, Ganjkar M, Miri Y, Fard MK (2016) The effect of two remineralizing agents and natural saliva on bleached enamel hardness. Dent Res J 13: 52.

15. Bowles WH, Ugwuneri Z (1987) Pulp chamber penetration by hydrogen peroxide following vital bleaching procedures. J Endod 13: 375-377.

16. Marson FC, Sensi LG, Vieira LCC, Araújo E (2008) Clinical evaluation of inoffice dental bleaching treatments with and without the use of light-activation sources. Oper Dent 33: 15-22.

17. Zach L, Cohen G (1965) Pulp response to externally applied heat. Oral Surg Oral Med Oral Pathol 19: 515-530.

18. Oliveira BP De, Bagnato VS, Panhoca VH (2017) SEM Analysis of a Peroxide Gel Whitening Protocol Associated to Light on Bovine Teeth. JSM Oro Facial Surgeries 2: 2-4.

19. Torres CRG, Perote LCCC, Gutierrez NC, Pucci CR, Borges AB (2013) Efficacy of mouth rinses and toothpaste on tooth whitening. Oper Dent 38: 57-62. 